\title{
Beyond biomarkers and towards functional outcomes for adult persons living with HIV. A cross sectional survey in Blantyre Urban, Malawi.
}

Steven Derrick Manyozo ( $\square$ derrickmanyozo@gmail.com )

University of Malawi College of Medicine

Nesto Tarimo

University of Malawi College of Medicine

Gift Kawalazira

Blantyre District Health Office

Adamson Sinjani Muula

University of Malawi College of Medicine

Research article

Keywords: HIV, disability, impairments, activity limitations, Blantyre, Malawi

Posted Date: December 5th, 2019

DOI: https://doi.org/10.21203/rs.2.18294/v1

License: (c) (i) This work is licensed under a Creative Commons Attribution 4.0 International License.

Read Full License 


\section{Abstract}

Introduction Despite improvements in survival, reports indicate that people living with HIV are experiencing a range of physical, cognitive, mental and social health-related challenges associated with HIV, comorbidities and aging recently termed 'disability'. Among adult persons living with HIV in Malawi, the prevalence of disability and associated risk factors are not known. This study was designed to assess the prevalence of impairments, activity limitations and associated risk factors among adults living with HIV in Blantyre urban, Malawi.

Methods We conducted a quantitative cross sectional study among adult persons living with HIV in Blantyre urban from March to August 2018. Participants were recruited consecutively from 5 health centers. We used the WHO's International Classification of Functioning, Disability and Health (ICF) for data collection. Written informed consent was sourced from study participants, permission was granted at each study site and the study received ethics approval from the College of Medicine Research Ethics Committee (COMREC). Descriptive statistics were used to summarize the data. Categorical variables were summarized as frequencies and percentages and continuous variables were summarized using means and standard deviation. Univariable and multivariable logistic regression were used to assess association between presence of disability and selected factors. Odds ratios (OR) and their $95 \%$ confidence intervals were calculated.

Results Of the 277 participants enrolled in the study, 225 (81\%) were female and 52 (19\%) were male. The mean age of participants was 37 years ( std. dev 9.5 ). Impairments in mental functions were the most prevalent affecting $118(43 \%)$ study participants. Energy and drive (18\%), memory (14\%) and sleep (13\%) were the most affected mental functions. Impairments in the mental functions, genital urinary, reproductive, digestive, metabolic and endocrine systems were associated with higher odds of disability among the participants.

Conclusions Our results indicate a high prevalence of impairments among persons living with HIV. Clinicians and rehabilitation personnel need to be proactive in identifying and managing impairments to promote health among HIV patients.

\section{Background}

The roll out and availability of antiretroviral therapy has changed the prognosis of HIV from what was inescapable death to a chronic manageable condition[1],[2]. Many of the estimated 35 million people currently living with HIV worldwide are expected to live almost near normal life expectancies[3]. While the rapid expansion in antiretroviral therapy access in low and middle income countries has resulted in dramatic declines in mortality rates, many people living with HIV face new or worsening experiences of disability[3].

Adequate management of HIV infection invites a shift to function and social participation outcomes and not only the clinical indices[4]. Despite improvements in survival, people with HIV are living with a range 
of physical, cognitive, mental and social health-related challenges, comorbidities and aging recently termed 'disability'[2],[5]. Using the international classification of function disability and health, reports from South Africa, Brazil and India highlight the high level of disablement and that up to $65 \%$ of HIV positive patients living with some form of body impairments[6],[7]. Findings from British Columbia show a high prevalence of disability among people with HIV exists, with over $80 \%$ having experienced at least one impairment, activity limitation or social participation restriction[2]. The implications of HIV-related disabilities are of global importance in HIV care especially in the era of improved longevity for HIV infected individuals [3][8][9].

Malawi aims to achieve the HIV 909090 goal, to diagnose $90 \%$ of all HIV-positive persons, provide antiretroviral therapy (ART) for $90 \%$ of those diagnosed, and achieve viral suppression for $90 \%$ of those treated by $2020[10],[11]$. By 2011, the country had $73 \%$ of those living with HIV knew their status, 86.2 of these are on treatment and about $90 \%$ of those on treatment have achieved viral suppression, many HIV infected individuals are therefore expecting to live near normal life expectancies[12],[13]. HIV is a significant challenge in Malawi, the revised National HIV Policy have highlighted the need to redefine strategies based on best evidence instituting multidisciplinary approach.[13],[14]. Recent evidence in Malawi suggests higher prevalence of disabilities among pediatric HIV patients where Devendra et al[15] looking at impairments, activity limitations and participation restrictions, reported a 33\% disability prevalence among HIV positive children in Lilongwe compared to $7 \%$ in controls. Hrapcak et al[16] in 2016 reported that hearing loss was more common among pediatric HIV patients and this affected their social functioning and school participation. However, knowledge of prevalence of disability among the adult HIV population in Malawi are not available.

\section{Methods}

\section{Study design}

We conducted a quantitative cross sectional study among adult HIV positive patients across 5 public health facilities in Blantyre urban, Malawi.

\section{Study setting}

The study was conducted at 5 local public health facilities in Blantyre urban: Zingwangwa, Limbe, Ndirande, Bangwe, and Chilomoni. These clinics are normally managed by a clinical officer as the incharge, nurse, pharmacy technician, psychosocial counselor and expert clients. The services offered at these facilities include HIV testing and counselling, ART administration, adherence support services through psychosocial counselling and viral load monitoring.

\section{Study population and sample size estimation}


Participants were recruited into the study if they were living with HIV, were on ART at least for 3 months, aged 18 years and above, attending their clinic within Blantyre urban as outpatient and were able to understand the study aims and consent properly. Data were collected from March to August 2018. Study participants were excluded if they were hospitalized. Participants were recruited consecutively from each health facility. Sample size was estimated in STATA ver 14 (StataCorp, College Station, TX, USA), at an estimated $50 \%$ prevalence of disability among HIV positive patients, $90 \%$ power and significance level set at $5 \%$.

\section{Ethical considerations}

The study was reviewed and approved by the College of Medicine Research and Ethics Committee (COMREC), (certificate number P.04/18/2388). Permission to conduct the study was granted by the Director of Health and Social Services for Blantyre district. To ensure privacy and confidentiality, codes were used on the questionnaire instead of participants' names. informed consent was obtained from participants, and where participants were illiterate a witness was invited to help explain the purpose of the research project during informed consent process. Research assistants were trained on the purpose of the study and all ethical requirements for the study.

\section{Data collection and instrumentation}

We conducted semi structured interviews using the International Classification of Function, Disability and Health (ICF) checklist[17].The ICF offers a useful framework for studying disablement and health-related consequences of disease based on understanding body impairments, activity limitations and participation restrictions. Impairments are understood to be problems with physiological functioning or anatomical (e.g., organs, limbs) structure of the body. Activity limitations are defined as difficulties in executing a task or action. Finally, participation restrictions were defined as problems relating to involvement in life situations. The aim of the tool is to provide a unified, standard language and framework for the description of health and health-related states[17]. The ICF has been reported to be a reliable tool in assessing health, social and environmental challenges faced by persons living with HIV [6], [18]. Figure 1 shows the ICF framework indicating the interaction between a health condition, body functions and structure and contextual factors to produce a functional outcome.

\section{Figure 1: ICF showing interaction between Health, Function and Disability, WHO 2005}

\section{Data management and analysis}


Data were entered into Microsoft excel spreadsheet and then exported into STATA ver 14 (StataCorp, College Station, TX, USA) for data cleaning and analysis. Data were summarized using proportions for categorical variables and continuous variables were summarized using means and standard deviations. Univariate and multivariate logistic regressions were used to summarize associations and assess risk factors for activity limitations and participation restriction. Significance level was set at $5 \%(0.05)$.

\section{Results}

Out of the 277 participants who participated in the study, 225 (81\%) were female and 52 (19\%) were male. The mean age of participants was 37 years (std. dev 9.5) with the youngest being 18 and the oldest participant was 78 years old. A total of $189(68 \%)$ were married and only $40(14 \%)$ were in formal employment. The majority of the participants $(126,45 \%)$ had primary school level training followed by secondary school $(111,40 \%)$. Table 1 summarizes patient socio-demographic characteristics.

\section{Table 1: Socio-demographic characteristics of study participants, Blantyre, Malawi N = 277}

\section{Impairments of body functions}

Table 2 presents a summary of impairments in body functions. Mental functions affected $118(43 \%)$ patients, energy and drive (18\%), memory (14\%) and sleep (13\%) were the most affected mental functions. About $40 \%$ of the participants reported having sensory problems, pain experience $(20 \%)$ and vestibular functions (19\%) were some of the most affected.

\section{Table 2: impairments of body functions disintegrated by system, Blantyre, Malawi N = 277}

\section{Activity limitations}

We found low levels of activity limitations and participation restriction among the people living with HIV. Interpersonal interactions (4\%) including intimate relationships and family relationships and mobility (3\%) were the most affected domains. Domains of general tasks and self- care were not affecting any of the participants. Table 3 presents a summary of the activity limitations and participation restrictions by domains.

\section{Table 3: Activity limitations and participation restrictions among people living with HIV, proportion affected by domain, $\mathrm{N}=277$}




\section{Associations between impairments of body functions and activity limitations}

We assessed impairments associated with mobility among the study participants. All impairments of body functions individually were associated with increased odds of reporting mobility problems. People living with HIV who had impairments in mental functions ( $A O R=7.97, p=0.06)$ and genitourinary and reproductive functions $(A O R=6.02, p=0.05)$ were associated with a significant increase in mobility problems. Affecting about $4 \%$ of the study population, interpersonal interactions and relations limitations were assessed on a logistic model to identify significant factors associated with the limitations in this domain. People living with impairments in the functions of the digestive, metabolic and endocrine systems were associated with increased limitations ( $A O R=6.37, p=0.01)$ in interpersonal interactions and relations Table 4 presents this summary.

\section{Discussion}

Our study highlights the high level of impairments of up to $43 \%$ among persons living with HIV. However, very few people reported activity limitations which were mainly in the area of mobility (3\%) and interpersonal interactions and relationships (4\%). Our findings indicate that as with many chronic conditions, rehabilitation will become an important part of HIV care[6]. High prevalence of impairments of body functions and variability reported in this study were also reported other studies[5],[6],[19]. Mental functions (43\%), sensory functions and pain (40\%) and functions of the cardiovascular, hematological, immunological and respiratory systems $(24 \%)$ were the most affected body functions. Mental function impairments, sleep disturbances, lack of energy and drive were very common among the study participants. Similar results have also been reported by Myezwa et al[6] in a South African HIV outpatient population, however our results indicate an average of $20 \%$ less impairments compared to those reported in South Africa. Furthermore, among our study participants, pain experience $(20 \%)$ and vestibular functions impairments (19\%) were some of the most reported impairments of body functions. These results highlight the need to comprehensively review and manage HIV patients beyond the routine HIV markers of viral load, CD4 count to including individual functional assessments.

Digestion (11\%) and weight maintenance (13\%) were the most common reported impairments within the domain of the functions of the digestive, metabolic and endocrine systems which was prevalent in $25 \%$ of our study participants. However, Myezwa et al in 2008[6] reported 2 times more impairments at $44 \%$ prevalence related to this domain among South African outpatient HIV positive patients. Myezwa et al in 2009[7] further reported among HIV positive inpatients 3 times more impairments (84\%) related to functions of digestive, metabolic and endocrine systems. This variation could be explained by the selfreport illness and disability experience as the concept of illness varies depending on how the individual understands it[20]. These variations in proportions affected are reflected in impairments related to the Neuromusculoskeletal systems, Myezwa et al in 2009 reported a $74 \%$ prevalence among HIV positive in patients and $27 \%$ prevalence among outpatients compared to the $18 \%$ reported in our study. 
Compared to impairments of body functions, activity limitations and participation restrictions were less common among persons living with HIV attending out-patient clinics in urban Blantyre. In our study, interpersonal interactions and relations (4\%) and mobility (3\%) were the most reported activity limitations and participation restriction. Mental function impairments, genitourinary and reproductive functions impairments and functions of the digestive, metabolic and endocrine systems impairments were the significant body impairments associated with the limitations in functions indicated above. A similar study conducted among children at the Malawi Baylor College of Medicine reported a higher prevalence of disability among HIV positive children (33\%) compared to HIV negative control (7\%), however it is important to note that most of the children in this study were also reported to have comorbid conditions that may have influenced the finding[15].

Despite the variations in prevalence of disability on different domains from different settings, our estimates are relatively low compared to those reported in India and South Africa[6],[7],[21]. A recent assessment of disability in a cohort of people living with HIV in South Africa and United States using the WHODAS questionnaire reported a $52 \%$ prevalence of disability among people living with HIV compared to what we are reporting in this study[22]. Across all these settings, the variability is high in terms of impairments in body functions and activity limitations and participation restrictions, measurement differences within the individual studies need to be considered as the studies used different tools, setting of the studies need to also be considered as it varies significantly in terms of health services organisation and patient socio-demographic profiles all of which may affect the individual study estimates. We have noted a growing adult population living with HIV among our study participants, mean, 37 years (std. dev 9.5) and the oldest was at 78 years old. These results agree with estimates reported from recent studies which have seen a significant increase in the number of older adults living with HIV[23][24][25].

Our study adds to the body of knowledge for HIV care in Malawi highlighting emerging issues to improve care for people living with HIV. It further highlights the need to look beyond limited clinical markers like CD4 counts, viral load and symptoms of HIV disease towards a comprehensive functional assessment and management to improve quality of life among HIV patients. The findings agree with findings from the HIV disability study conducted among children living with HIV in Lilongwe, Malawi where a high magnitude of disability was reported demonstrating the need for rehabilitation services among people living with HIV in the era of improved longevity.

At macro level, HIV policy implications are important to also consider. The Malawi national strategic plans recognize that HIV remains a significant challenge in the country and requires a revision of strategies based on both emerging and best evidence instituting multidisciplinary approach towards HIV response[26]. Similarly, with the emergency of HIV long terms survivors, the South African national strategic plan on HIV/TB/STI 2017-2022 duly recognize the need to address health needs of this population and have included rehabilitation as a critical component in HIV care[27]. The aging HIV population with new disability experiences and impairments will require particular attention to address chronic HIV syndrome in the face of comorbidities that increase with age such as hypertension and diabetes[28]. Recently, population level interventions including exercise have become popular 
interventions to avert some of the HIV associated impairments and limitations. Researchers in Canada are evaluating the impact of community based exercise program with the aim of reducing disability and enhancing health living[22].

\section{Study Limitations}

One of the limitations to our study is that we did not consider the effect of ART regimen which could potentially affect the presence or absence of body impairments and activity limitations. We did not also collect data on co-morbidities which could further help explain the impairments and disability phenomenon presented in this paper. Further research should assess these important factors and their role in disability experience among patients with chronic HIV infection. There is also need to review evidence for health promotion among HIV survivors. Finally, the study was facility based which may have been affected by selection bias in favor of those that only made it to the clinics during the survey time.

\section{Conclusion And Recommendations}

Our study highlights the high level of impairments of up to $43 \%$ among persons living with HIV. However, very few people reported activity limitations which were mainly in the area of mobility (3\%) and interpersonal interactions and relationships (4\%). The problems of pain, weight maintenance, lack of sleep and energy and drive and their subsequent effects on activity limitations reported among the participants need to be considered in the continuum of care for HIV patients. Clinicians and rehabilitation personnel need to be proactive in identifying and managing impairments among people living with HIV.

\section{Declarations}

\section{Author contributions}

SM, TN, ASM conceived the project, data collection was led by SM. SM in consultation with TN, ASM and GK analysed the data. All authors contributed to the development and revision of the manuscript and approved the final version.

\section{Acknowledgements}

We would like to thank the Africa Center of Excellence in Public Health and Herbal Medicine for the grant and technical guidance during study implementation. We would also like to appreciate the study participants who freely volunteered to take part.

\section{Ethics declarations}

\section{Ethical approval and consent to participate}


The study proposal was reviewed and approved by the College of Medicine Research Ethics Committee (COMREC) on May 29, 2018 - (certificate number P.04/18/2388). Written Informed consent was collected before conducting any study related procedures.

\section{Consent for publication}

Not applicable

\section{Competing interests}

Adamson Muula is a member of the editorial board for the BMC public health journal, otherwise the rest of the members declare no conflicting interests.

\section{Availability of data for the study}

The datasets used and/or analysed during the current study are available from the corresponding author on reasonable request

\section{Funding for the study}

The study was funded by the Africa Center of Excellence in Public Health and Herbal Medicine (ACEPHEM). ACEPHEM is a World Bank funded project under the Africa Centers of Excellence phase II (ACEII) and is based at the University of Malawi's College of Medicine.

\section{References}

1. Banks LM, Zuurmond M, Ferrand R, Kuper H. Knowledge of HIV-related disabilities and challenges in accessing care: Qualitative research from Zimbabwe. PloS one. 2017 Aug 9;12(8):e0181144.

2. O'Brien KK, Ibáñez-Carrasco F, Solomon P, Harding R, Cattaneo J, Chegwidden W, Gahagan J, Baxter L, Worthington C, Gayle P, Merritt B. Advancing research and practice in HIV and rehabilitation: a framework of research priorities in HIV, disability and rehabilitation. BMC infectious diseases. 2014:14(1):724.

3. Nixon SA, Hanass-Hancock J, Whiteside A, Barnett T. The increasing chronicity of HIV in sub-Saharan Africa: Re-thinking" HIV as a long-wave event" in the era of widespread access to ART. Globalization and health. 2011;7(1):41.

4. O'brien KK, Bayoumi AM, Strike C, Young NL, Davis AM. Exploring disability from the perspective of adults living with HIV/AIDS: development of a conceptual framework. Health and Quality of Life Outcomes. 2008;6(1):76. 
5. Rusch M, Nixon S, Schilder A, Braitstein P, Chan K, Hogg RS. Impairments, activity limitations and participation restrictions: prevalence and associations among persons living with HIV/AIDS in British Columbia. Health and quality of life outcomes. 2004;2(1):46.

6. Van As M, Myezwa H, Stewart A, Maleka D, Musenge E. The International Classification of Function Disability and Health (ICF) in adults visiting the HIV outpatient clinic at a regional hospital in Johannesburg, South Africa. Aids Care. 2009;21(1):50-8.

7. Myezwa H, Stewart A, Musenge E, Nesara P. Assessment of HIV-positive in-patients using the international classification of functioning, disability and health (ICF) at Chris Hani Baragwanath Hospital, Johannesburg. African Journal of AIDS Research. 2009;8(1):93-105.

8. World Health Organization (WHO). Progress Report 2016, prevent HIV, test and treat all. Who. 2016;64.

9. Jung A, Raman A, Mallewa M, Solomon T. Neurology research and teaching in Malawi. Clinical medicine. 2009 Dec 1;9(6):570-1.

10. UNAIDS. Ending AIDS: Progress Towards the 90-90-90 Targets. 2017;6-21.

11. Government of Malawi. Malawi AIDS Response Progress Report. 2015:1-79.

12. Dasgupta AN, Wringe A, Crampin AC, Chisambo C, Koole O, Makombe S, Sungani C, Todd J, Church K. HIV policy and implementation: a national policy review and an implementation case study of a rural area of northern Malawi. AIDS care. 2016;28(9):1097-109.

13. Government of Malawi. Ministry of Health. National Strategic Plan for HIV and AIDS 2011-15.

14. Government of Malawi. National Strategic Plan for HIV and AIDS 2015 - 2020.

15. Devendra A, Makawa A, Kazembe PN, Calles NR, Kuper H. HIV and childhood disability: a casecontrolled study at a paediatric antiretroviral therapy centre in Lilongwe, Malawi. PloS one. 2013;8(12):e84024.

16. Hrapcak S, Kuper H, Bartlett P, Devendra A, Makawa A, Kim M, Kazembe P, Ahmed S. Hearing loss in HIV-infected children in Lilongwe, Malawi. PloS one. 2016;11(8):e0161421.

17. Kostanjsek N. Use of The International Classification of Functioning, Disability and Health (ICF) as a conceptual framework and common language for disability statistics and health information systems. BMC public health 2011:11 (4).

18. Cobbing S, Hanass-Hancock J, Myezwa H. A home-based rehabilitation intervention for people living with HIV and disability in a resource-poor community, KwaZulu-Natal: study protocol for a randomised controlled trial. Trials. 2015;16(1):491.

19. Hwang JL, Nochajski SM. The International Classification of Function, Disability and Health (ICF) and Its Application with AIDS. Journal of Rehabilitation. 2003;69(4).

20. Wikman A, Marklund S, Alexanderson K. Illness, disease, and sickness absence: an empirical test of differences between concepts of ill health. Journal of Epidemiology \& Community Health. 2005;59(6):450-4. 
21. Gaidhane AM, Zahiruddin QS, Waghmare L, Zodpey S, Goyal RC, Johrapurkar SR. Assessing self-care component of activities and participation domain of the international classification of functioning, disability and health (ICF) among people living with HIV/AIDS. AIDS care. 2008;20(9):1098-104..

22. Myezwa H, Hanass-Hancock J, Ajidahun AT, Carpenter B. Disability and health outcomes-from a cohort of people on long-term antiretroviral therapy. SAHARA-J: Journal of Social Aspects of HIV/AIDS. 2018;15(1):50-9.

23. Mavandadi S, Zanjani F, Ten Have TR, Oslin DW. Psychological wellbeing among individuals aging with HIV: The value of social relationships. Journal of acquired immune deficiency syndromes (1999). 2009;51(1):91.

24. Cardoso SW, Torres TS, Santini-Oliveira M, Marins LM, Veloso VG, Grinsztejn B. Aging with HIV: a practical review. The Brazilian Journal of Infectious Diseases. 2013;17(4):464-79.

25. Centers for Disease Control and Prevention. HIV Surveillance Report, 2016;28.

26. Government of Malawi. National HIV and AIDS policy. 2011 - 2016.

27. South African National AIDS Council. Let our actions count: South Africa's national strategic plan for HIV, TB and STIs 2017-2022.

28. Slomka J, Lim JW, Gripshover B, Daly B. How have long-term survivors coped with living with HIV?. Journal of the Association of Nurses in AIDS Care. 2013;24(5):449-59.

\section{Tables}

Table 1

\begin{tabular}{llll}
\hline Variable & Attribute & \multicolumn{2}{l}{ Frequency, n (\%) } \\
\hline Gender & Male & 52 & $(19 \%)$ \\
& Female & 225 & $(81 \%)$ \\
\hline Age (mean, 37 years (std. dev 9.5$)$ & $<25$ & 34 & $(12 \%)$ \\
\hline & $\geq 25$ & 243 & $(88 \%)$ \\
\hline Marital status & Single & 88 & $(32 \%)$ \\
\hline Educational status & Married & 189 & $(68 \%)$ \\
\hline & None & 17 & $(6 \%)$ \\
\hline & Primary & 126 & $(45 \%)$ \\
\hline Occupation status & Secondary & 111 & $(40 \%)$ \\
\hline & Tertiary & 23 & $(8 \%)$ \\
\hline & Formal employment & 40 & $(14 \%)$ \\
\hline
\end{tabular}




\section{Table 2}

\begin{tabular}{lcccc}
\hline & \multicolumn{2}{c}{$\begin{array}{c}\text { Persons experiencing } \\
\text { impairments }\end{array}$} & $\begin{array}{c}\text { Persons not experiencing } \\
\text { impairments }\end{array}$ \\
\cline { 2 - 5 } & \multicolumn{2}{c}{$\mathrm{N}(\%)$} & $\mathrm{N}(\%)$ \\
\hline unctions & 118 & $(43 \%)$ & 159 & $(57 \%)$ \\
functions and pain & 111 & $(40 \%)$ & 166 & $(60 \%)$ \\
1s of the cardiovascular, hematological, & 66 & $(24 \%)$ & 211 & $(76 \%)$ \\
logical and respiratory systems & & & & \\
1s of the digestive, metabolic and endocrine & 70 & $(25 \%)$ & & \\
& & & 207 & $(75 \%)$ \\
rinary and reproductive functions & 17 & $(6 \%)$ & 227 & $(82 \%)$ \\
\hline usculoskeletal and movement-related functions & 50 & $(18 \%)$ & 246 & $(89 \%)$ \\
\hline 1s of the skin and related structures & 31 & $(11 \%)$ & & \\
\hline
\end{tabular}

Table 3

\begin{tabular}{lllc}
\hline & $\begin{array}{l}\text { Participants with activity } \\
\text { limitations }\end{array}$ & \multicolumn{2}{l}{$\begin{array}{l}\text { Participants without activity } \\
\text { limitations }\end{array}$} \\
\cline { 2 - 4 } & $\mathrm{N}(\%)$ & $\mathrm{N}$ & $(\%)$ \\
\hline y and applying knowledge & $2(0.7 \%)$ & 275 & $(99.3 \%)$ \\
tasks and demands & 0 & 270 & $(100 \%)$ \\
\hline & $7(3 \%)$ & 277 & $(100 \%)$ \\
Ire & 0 & 273 & $(98.6 \%)$ \\
c life & $4(1.4 \%)$ & 266 & $(96 \%)$ \\
sonal interactions and & $11(4 \%)$ & & \\
ships & & 277 & $(100 \%)$ \\
\hline fe areas & 0 & 275 & $(99.3 \%)$ \\
\hline
\end{tabular}

Figures 
Figure 1

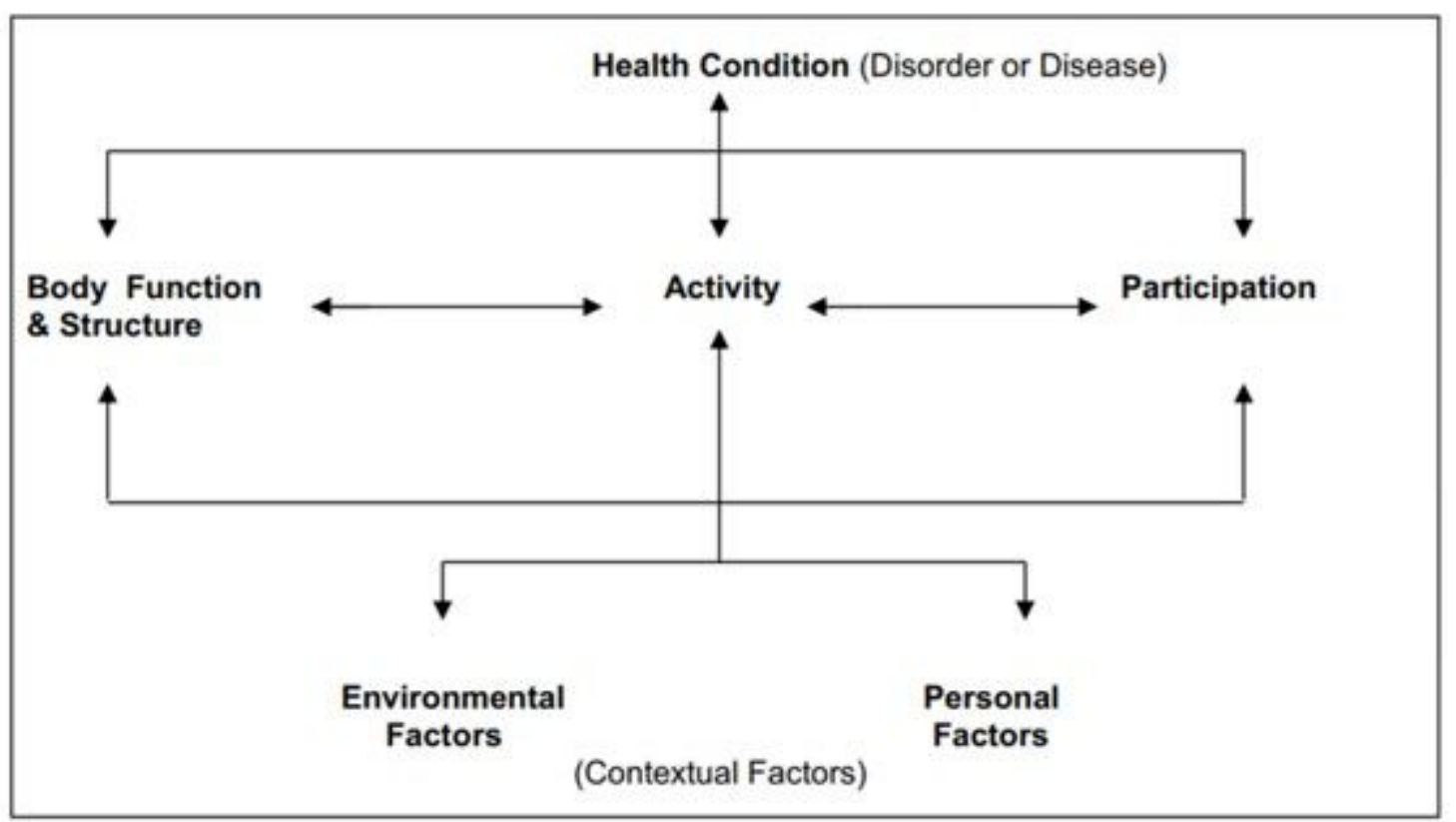

Figure 1

ICF showing interaction between Health, Function and Disability, WHO 2005 